\title{
Sampling-induced artifacts in incubation experiments on biological processing of dimethylsulfide and similarly soluble gases
}

\author{
Richard W. Hill ${ }^{1, *}$, John W. H. Dacey ${ }^{2}$ \\ ${ }^{1}$ Department of Zoology, Michigan State University, East Lansing, Michigan 48824, USA \\ ${ }^{2}$ Department of Biology, Woods Hole Oceanographic Institution, Woods Hole, Massachusetts 02543, USA
}

\begin{abstract}
Aquatic biological systems that produce or transform dimethylsulfide (DMS), methanethiol, and similarly soluble gases are often studied by means of incubation experiments in closed bottles containing liquid and gas phases. During such experiments, liquid samples are often removed sequentially to obtain time-series of data on microbial numbers, concentrations of nongaseous compounds, or other liquid-phase parameters. The removal of liquid samples from closed bottles affects the concentrations in both liquid and gas phases of 2-phase compounds such as DMS, because sampling alters the liquid:gas volume ratio, inducing chemical redistribution. Three types of problems can arise from multiple sampling in studies of volatile compounds: (1) standardization may become inaccurate because sampling may render experimental and standard bottles incommensurate by creating unequal liquid:gas ratios in the 2 sorts of bottles; (2) changes in concentrations in experimental bottles caused by biological processes may be confounded by sampling-induced changes in concentrations; and (3) concentrations of standards may be altered by sampling from standard bottles. Mathematical expressions of all 3 types of potential distortion caused by sampling are possible, and are useful both for error correction and for designing experiments to minimize artifacts. Mathematical solutions indicate that of the 3 problems, the first is the one that can introduce the quantitatively greatest artifacts. Problem (2) is the most common concern because at least a small confounding of biological and sampling-induced concentration changes is inevitable; the problem is more likely to introduce quantitatively consequential artifacts during falling gas concentrations than rising ones, according to mathematical analysis. The mathematical solutions to Problems (1) and (2) depend on whether the biological cultures are homogeneous. The magnitudes of all 3 problems depend on the partitioning behavior of the gas studied; for instance, in a series of gases that partition principally into the liquid phase, the potential for artifact increases as solubility decreases.
\end{abstract}

KEY WORDS: Incubation studies $\cdot$ Henry's law $\cdot$ Ostwald coefficient $\cdot$ Biogenic gases $\cdot$ Dimethylsulfide

\section{INTRODUCTION}

Investigations of aquatic biological systems that are sources or sinks for atmospheric gases are often carried out on cultures in closed vessels. Biological systems thus studied to better understand dimethylsulfide (DMS), for example, include cultures of algae (e.g., Stefels \& van Boekel 1993), algae and viruses (e.g., Hill et

\footnotetext{
*E-mail: hillr@msu.edu
}

al. 1998, Malin et al. 1998), bacteria (e.g., Bak et al. 1992, Ledyard \& Dacey 1996), algae and heterotrophic protists (e.g., Wolfe \& Steinke 1996, Wolfe et al. 1997), algae and zooplankton (e.g., Dacey \& Wakeham 1986, Daly \& DiTullio 1996), and natural microbial assemblages (e.g., Kiene \& Service 1991, Visscher et al. 1994, Kiene 1996). During studies of such systems in closed vessels, it is often desired to withdraw multiple liquid samples from the vessels over time to obtain time-series of data on microbial numbers, concentrations of non- 
gaseous compounds, or other liquid-phase parameters. A headspace usually exists for physiological or practical reasons. This paper concerns artifacts that can arise from the removal of multiple liquid samples from closed vessels containing both gas and liquid phases. Entities confined to the liquid phase, such as nongaseous solutes and microbial populations, are not at issue; the removal of a liquid sample does not alter their concentrations or densities. Instead, our concern here is with compounds that partition between the gas and liquid phases, such as DMS. Each removal of a liquid sample itself alters the concentrations in both the gas and liquid phases of such compounds, because the withdrawal of liquid changes the liquid:gas volume ratio within a study bottle, inducing redistribution of a 2-phase compound. Sampling in such cases can significantly affect parameters of such fundamental importance as the relation between experimental bottles and standards.

Compounds that exist in both gas and liquid phases exhibit a broad spectrum of partitioning behavior. The dimensionless Ostwald coefficient $L$ is one measure of partitioning, defined to be the ratio of the concentration in the liquid phase over the concentration in the gas phase at equilibrium (Clever \& Battino 1975, Gerrard 1980). The coefficient is temperature dependent. A compound with a very high Ostwald coefficient exists almost entirely in the liquid phase and behaves much like a nongaseous solute, whereas the reverse is true of a compound with a very low $L$. Our concern here is with compounds of intermediate $L$, having a substantial presence in both phases. Limits of relevant $L$ are not simply demarcated. Our focus is on $L=2-20$. Partitioning in this range is exhibited by a variety of sulfur gases, many of which are products of or subject to biological processing: not only DMS but also dimethyldisulfide, several dialkylsulfides, methanethiol, and other thiols (Przyjazny et al. 1983). Nonsulfur compounds that partition in the relevant range and are of similar biological interest include certain monoaromatic compounds (e.g., benzene and toluene), halogenated monoaromatics, and halogenated alkenes or alkanes (e.g., chloromethane) (Mackay \& Shiu 1981). Understanding biological processing that affects the ecological and biogeochemical dynamics of gases such as these will inevitably invite study by the sort of multiple-sample incubation experiments that are the focus of this paper.

Our attention was drawn to these issues by studies of DMS, and we use DMS as our focal example. DMS has an Ostwald coefficient of about 11 at $22^{\circ} \mathrm{C}$ in a seawater:air system (Przyjazny et al. 1983, Dacey et al. 1984). DMS is of great current interest because in the atmosphere its oxidation products affect cloud cover over the oceans and, thus, global climate (Andreae \& Crutzen 1997). DMS is formed from the nonvolatile precursor dimethylsulfoniopropionate (DMSP), which is synthesized principally by phytoplankton in ocean surface waters. The formation of DMS from algal intracellular DMSP is promoted by algal and bacterial enzymatic degradation of DMSP, grazing by minute phytoplanktivores, algal senescence, and viral lysis of algal cells (Malin \& Kirst 1997, Hill et al. 1998). DMS is catabolized by seawater microbes (e.g., Kiene \& Service 1991). The great diversity of incubation studies on DMS noted at the start of this report reflects the effort to understand the wide range of aquatic biological processes that interact dynamically to set [DMS] in ocean surface waters and thereby affect outgassing of DMS to the atmosphere.

We present mathematical solutions to 3 problems that arise in studies of volatile compounds $(L=2-20)$ during incubation experiments in which multiple liquid samples are removed from bottles. The first is a relational problem between experimental bottles and standards, arising because sampling can shift the relative liquid:gas volume ratios in the 2 types of bottles. The second and third problems we address arise from the fact that sampling alters concentrations within individual bottles; thus, on the one hand, sampling from an experimental bottle can confound biologically mediated changes in concentration in the bottle, and on the other hand, sampling from a standard bottle can alter the accuracy of the standard. Although we address only these specific problems, our analytical approach has obvious applications to a number of other issues. One of our overriding goals is to show how experimental results on volatile compounds should be calculated to take account of sampling effects. A second major goal is to aid experimental design by articulating the quantitative implications of factors that are under experimental control.

To assess the need for the sort of analysis we present, we have reviewed the literature on incubation experiments on DMS. Some papers on DMS also report on methanethiol or other compounds with relevant $L$, but for the most part we have not looked at the literature on other compounds besides DMS. If we had, we presume our list of examples would be greatly expanded.

A disturbing initial discovery from reviewing the literature was that among the ca 20 incubation studies we found that involved multiple sampling from experimental bottles, at least 6 studies, published in prominent journals since 1992, either omit all volumetric details of bottle set-up and sampling or fail to report some such details required for a reader to gauge the likelihood of sampling-induced artifacts. The potential artifacts we discuss depend on liquid:gas volume ratios in incubation bottles and on sampling-induced shifts in the ratios, which depend on the numbers of samples removed and on sample volumes relative to bottle vol- 
umes. These details have as great a potential to introduce artifacts as details of analytical chemistry, reagent purity, microbial enumeration, or filtration. Yet, whereas details of the latter sorts are routinely viewed as essential content for a research report, papers on multiple-sample incubation studies often omit critical volumetric details. This failure to report critical information suggests inadequate appreciation of the potential artifacts that can be induced by sampling in studies of volatile compounds. At the least, it leaves us and other readers unable to reach an independent judgement about the appropriateness of methods used.

Among the subset of papers on multiple-sample incubation studies that specify all the necessary details (liquid:gas volume ratios in bottles, effects of sampling on the ratios, etc.), we found not one paper that acknowledges that those details matter for the estimation and management of potential sampling-induced artifacts. This finding again suggests that the possibility of artifacts from sampling is inadequately appreciated. More important, in some of these papers, the investigators report sampling protocols that unwittingly but almost certainly introduced errors in results. For example, 2 papers from 2 different laboratories report protocols that removed so much liquid from incubation bottles that the gas space in the bottles increased by at least 20 to $25 \%$ of bottle volume during experiments. Two other papers report that the gas space potentially increased by $10 \%$ or more of bottle volume while [DMS] was declining. As we later show, sampling protocols of these sorts invite substantial sampling-induced artifacts.

Investigators carrying out incubation studies have sometimes used 1 of 2 designs that keep liquid:gas ratios constant in bottles and thereby avoid samplinginduced shifts of gas partitioning. One design that achieves this end is to start with no headspace and replace removed water so no headspace forms. We found this design used once in the DMS literature. Our concern with it is that although many consequences of the water additions can in principle be calculated, the additions may have significant nonquantifiable effects (e.g., on nutrient concentrations and density-dependent microbial interactions). The second design that avoids consequences of multiple sampling is to sample bottles only once and use multiple bottles to establish the time-series. Thus, instead of sampling each replicate bottle on $T$ occasions, $T$ times as many bottles are set up at the beginning of an experiment and each is sampled only once. We found this design used in 5 papers. It assumes that sets of truly identical bottles can be produced at the start and will follow identical trajectories. Our experience is that inter-bottle variation is common. Inter-bottle variation becomes confounded with time-dependent effects in the design.
Substantial temporal discontinuities, presumed to arise artifactually in this way, are evident in 2 of the 5 reports using the design.

We propose that the inherently best design for timeseries experiments in most cases is the repeated sampling of bottles without water replacement, because this design is most likely to minimize extraneous or confounding biological effects. However, for the design to rest on a firm conceptual foundation, sampling effects must be addressed, and analytical solutions of sampling effects are needed. This paper aims to correct the present deficiency.

As earlier noted, we found ca 20 studies in the literature on DMS that used the repeated-sampling design, but about half either did not fully report volumetric details of protocols or employed protocols that almost surely introduced artifacts. We do not assert that sampling effects in prior research ever led to qualitatively incorrect conclusions, but needless quantitative errors seem likely in some studies.

\section{ANALYTICAL SOLUTIONS}

\section{Problem 1: Relation between experimental bottles and standards}

The relation between experimental bottles and standards is the quantitatively most substantial problem that must be addressed in multiple-sample bottle experiments. Suppose that the fraction of total volume filled with liquid is initially the same in both experimental bottles and standard bottles. Removal of liquid samples can cause the 2 types of bottles to become mismatched in this regard as a study proceeds. DMS concentrations in the experimental and standard bottles will then cease to be immediately commensurate because the partitioning of DMS between liquid and gas depends on the liquid:gas volume ratio. Standardization will be inaccurate unless the problem is addressed.

Table 1 presents variables that describe a sealed bottle. To clarify the practical application of our analysis, we mention details of recent experiments we performed (Hill et al. 1998). In each experimental bottle, which had a total volume $\left(V_{\mathrm{T}}\right)$ of $1070 \mathrm{ml}$, we placed $700 \mathrm{ml}$ of an algal culture in seawater, inoculated with virus. Thus, the fraction of bottle volume filled with liquid $\left(F_{V(l)}\right)$ was initially 0.65 . We periodically removed $14 \mathrm{ml}$ of culture for analysis. Ten samples reduced $F_{V(1)}$ stepwise to 0.52 .

Our standards were bottles partly filled $\left(F_{v(l)}=0.65\right)$ with seawater of stated [DMS]. DMS added to create a standard was calculated in reference to liquid volume, as is common practice. For example, to make a $100 \mathrm{nM}$ 
Table 1. Symbols referring to properties of sealed bottles containing gas and liquid phases, plus other symbols used. DMS serves as the focal example of a volatile compound in this paper. Other compounds can be substituted for DMS

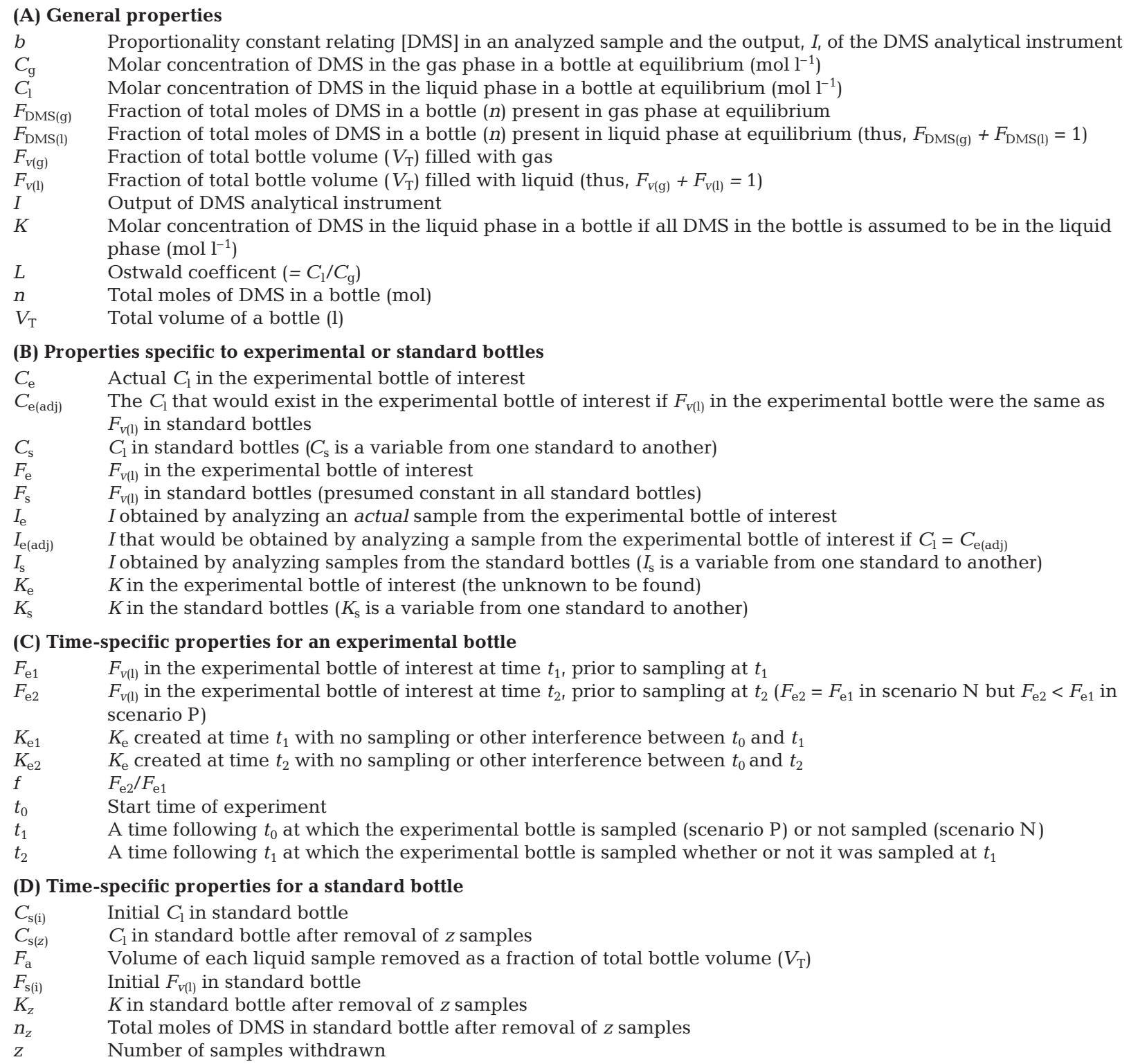

standard, $10 \mathrm{nmol}$ of DMS was added to $100 \mathrm{ml}$ of liquid. Note that although the liquid [DMS] was truly $100 \mathrm{nM}$ at the moment we made this solution, it was only nominally $100 \mathrm{nM}$ after equilibration with gas phase in the standard bottle. At equilibrium, neither the liquid nor the gas in the '100 nM' standard in fact had $[\mathrm{DMS}]=100 \mathrm{nM}$.

Henry's law provides a simple resolution of the problem presented by the just-noted discrepancy if $F_{V(l)}$ is identical in standard and experimental bottles, because it predicts that then the proportion of DMS in the liquid phase, $F_{\mathrm{DMS}(1)}$, is also identical in the 2 sorts of bot- tles (see Eq. 5). Thus, an experimental bottle with the same liquid [DMS] as a nominal $100 \mathrm{nM}$ standard would, like the standard, contain total DMS exactly sufficient to make the liquid $100 \mathrm{nM}$ if all DMS were in the liquid.

This simple resolution of the problem does not apply if $F_{V(l)}$ differs between experimental and standard bottles. In our study, if $F_{V(1)}$ was 0.65 in standards but 0.52 in experimental bottles because of sampling, DMS partitioning between liquid and gas would be disproportionate in the two. Thus, the quantitative meaning of a similarity in liquid [DMS] between a standard bottle and an experimental bottle would be confused. 
For some chemicals, the only answer to the standardization problem might be an experimental design that physically keeps $F_{V(l)}$ identical in experimental and standard bottles. However, an analytical solution exists for many chemicals.

We assume that the culture constituting the liquid phase in an experimental bottle is homogeneous, meaning operationally that (1) the mix of living organisms is assumed to be functionally identical in each unit volume of experimental relevance and (2) sample removal is assumed (on average) to deplete all types of organisms in equal proportion. The assumption of homogeneous cultures is likely to be accurate in essentially all work on microbial systems. We briefly mention nonhomogeneous cultures at the end. We also assume: (1) Experimental bottles and standards are isothermal and matched in solutes (e.g., salts) that affect DMS phase partitioning. (2) The Ostwald relation $L=C_{\mathrm{l}} / C_{\mathrm{g}}$ applies (Clever \& Battino 1975, Gerrard 1980). (3) The output $I$ of the DMS analytical instrument is proportional to actual [DMS] in analyzed samples or has been converted to a proportional scale: $I=b$ [DMS], where $b$ a constant. Failure of assumption (3) would not negate our approach but would necessitate additional mathematical manipulations.

To introduce our analysis, we assume also that the chemical of interest originates entirely from the liquid phase. Experimental bottles meet this assumption in studies of DMS formation because aquatic organisms are the source of DMS. For standards to meet the assumption, they must be prepared as described earlier: The liquid is first brought to the stated [DMS] of the standard and then allowed to equilibrate with a gas phase that previously was devoid of DMS. Let $K$ be liquid [DMS] within a bottle when all DMS is presumed to be in the liquid:

$$
K=n /\left(F_{V(1)} V_{\mathrm{T}}\right)
$$

Thus, $K$ for a standard bottle is the nominal [DMS] of the standard, and $K$ for an experimental bottle is the [DMS] the organisms would create in the water were all DMS to stay in the water.

If liquid with concentration $K$ is placed in a bottle initially devoid of DMS, $n=K F_{V(1)} V_{\mathrm{T}}$ according to Eq. (1), and [DMS] in the gas phase after equilibration is:

$$
C_{\mathrm{g}}=\frac{n F_{\mathrm{DMS}(\mathrm{g})}}{V_{\mathrm{T}} F_{V(\mathrm{~g})}}=\frac{K F_{V(1)} F_{\mathrm{DMS}(\mathrm{g})}}{F_{V(\mathrm{~g})}}
$$

A similar derivation yields:

$$
C_{1}=K F_{\mathrm{DMS}(1)}
$$

Substituting Eqs. (2) \& (3) in the Ostwald relation, we get the following expression that pertains in the bottle at equilibrium:

$$
\frac{F_{\mathrm{DMS}(\mathrm{g})}}{F_{V(\mathrm{~g})}}=\frac{F_{\mathrm{DMS}(1)}}{L F_{V(1)}}
$$

Recognizing that $F_{\mathrm{DMS}(\mathrm{g})}=1-F_{\mathrm{DMS}(\mathrm{l})}$, and $F_{v(g)}=1-F_{V(1)}$, Eq. (4) transforms to:

$$
F_{\mathrm{DMS}(1)}=\frac{L F_{V(1)}}{1+(L-1) F_{V(1)}}
$$

Combining Eqs. (3) \& (5), we get the following pivotal expression that describes the relation between [DMS] in the liquid phase, $C_{\mathrm{l}}$, and fractional liquid volume, $F_{V(1)}$, at equilibrium when all DMS is introduced by way of a liquid at initial concentration $K$ :

$$
C_{1}=\frac{K L F_{V(1)}}{1+(L-1) F_{V(1)}}
$$

Consider, now, the practical situation faced: One has an experimental bottle with a $F_{V(1)}$ that does not necessarily match that in standards. One also has a series of standards of various $K$ values, all made at one $F_{V(1)}$. Table 1B defines symbols, now to be used, that refer specifically to the experimental bottle or the standard bottles.

Analysis of the standards (using either gas or liquid phase) yields a proportional standard curve:

$$
I_{\mathrm{s}}=b K_{\mathrm{s}}
$$

We know $I_{\mathrm{e}}$ (assumed to be based on analysis of the same phase as the standard curve), and we desire to know $K_{\mathrm{e}}$. If and only if $F_{\mathrm{e}}=F_{\mathrm{s}}$, can $I_{\mathrm{e}}$ be entered into Eq. (7) to obtain $K_{\mathrm{e}}$. If $F_{\mathrm{e}}$ is unequal to $F_{\mathrm{s}}$ one approach is to modify $I_{\mathrm{e}}$ mathematically so that when it is entered into Eq. (7), the correct $K_{\mathrm{e}}$ is obtained. Another approach is to modify Eq. (7) to apply to $F_{V(1)}=F_{\mathrm{e}}$ and enter the actual $I_{\mathrm{e}}$. We present the former approach here, but both yield the same solution.

Our goal is to find a mathematical adjustment to $I_{\mathrm{e}}$ such that correct results will be obtained by using Eq. (7) to calculate $K_{\mathrm{e}}$. The key to our solution is to recognize that in a homogeneous culture, the DMS yielded by organisms per unit liquid volume, $K_{\mathrm{e}}$, can be expected to be the same whether $F_{V(1)}$ in the experimental bottle is $F_{\mathrm{s}}$ or $F_{\mathrm{e}}$. The actual molar concentration of DMS in the liquid phase of the experimental bottle at equilibrium, $C_{\mathrm{e}}$, is expressed by Eq. (6) if $K$ is set equal to $K_{\mathrm{e}}$ and $F_{V(1)}$ is set equal to the actual value in the experimental bottle, $F_{\mathrm{e}}$ :

$$
C_{\mathrm{e}}=\frac{K_{\mathrm{e}} L F_{\mathrm{e}}}{1+(L-1) F_{\mathrm{e}}}
$$

If the experimental bottle had the same $F_{V(1)}$ as standards, then the molar concentration of DMS in the liquid phase at equilibrium would be:

$$
C_{\mathrm{e}(\mathrm{adj})}=\frac{K_{\mathrm{e}} L F_{\mathrm{s}}}{1+(L-1) F_{\mathrm{s}}}
$$


Because of the proportionality of instrument output and concentration, the instrument output for $C_{\mathrm{e}(\mathrm{adj}) \text {, }}$

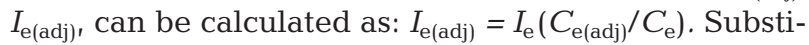
tuting Eq. (8) and Eq. (9):

$$
I_{\mathrm{e}(\mathrm{adj})}=I_{\mathrm{e}}\left(\frac{F_{\mathrm{s}}\left[1+(L-1) F_{\mathrm{e}}\right]}{F_{\mathrm{e}}\left[1+(L-1) F_{\mathrm{s}}\right]}\right)
$$

$I_{\mathrm{e}(\mathrm{adj})}$ is the instrument output to be expected from the experimental bottle if $F_{\mathrm{e}}$ were the same as the $F_{V(1)}$ in standards, $F_{\mathrm{s}}$. Thus, entering $I_{\mathrm{e}(\mathrm{adj})}$ in Eq. (7), a relation based on $F_{V(1)}=F_{\mathrm{s}}$ yields the correct $K_{\mathrm{e}}$. This is true whether gas or liquid samples are analyzed. Although we have focused on liquid samples in deriving Eq. (10), the equation is the same when gas samples are analyzed.

Fig. 1A presents 2 examples of the relation between the correction factor (multiplier of $I_{\mathrm{e}}$ ) in Eq. (10) and $F_{\mathrm{e}}$. The errors of ignoring differences between $F_{\mathrm{e}}$ and $F_{\mathrm{s}}$ are reflected by the values on the $y$-axis (e.g., a correction of 1.1 signifies an error of about $10 \%$ ). Note that poor design, without correction, can lead to severe errors. Informed design, however, can permit corrections to be limited to $5 \%$ or less, even while numerous samples are taken from an experimental bottle.

Rigorously, for our analytical solution to be applied, (1) standards must be prepared as specified, and (2) $K$

Fig. 1. Examples of distortions caused by sampling in the 3 problem areas addressed by this paper for Ostwald coefficient $L=11$. (A) The multiplier of $I_{\mathrm{e}}$ in Eq. (10), here termed the correction factor to match bottle types, as a function of fractional liquid volume in an experimental bottle $\left(F_{\mathrm{e}}\right)$ when using standard bottles having 2 different constant fractional liquid volumes $\left(F_{\mathrm{s}}\right)$. The correction factor is the factor by which the actual instrument output for a sample from the experimental bottle must be multiplied to obtain valid concentrations from the standard curve, derived from standard bottles, when $F_{\mathrm{e}} \neq$ $F_{\mathrm{s}}$. Note that the $F_{\mathrm{s}}$ employed (even a very high one) has little effect on sensitivity of the correction factor to $F_{\mathrm{e}}$ versus $F_{\mathrm{s}}$ differences. (B) Factorial distortion of $K_{\mathrm{e}}$ (total moles of DMS expressed as a ratio of liquid volume) in an experimental bottle caused by prior sampling as a function of $K_{\mathrm{e} 2} / K_{\mathrm{e} 1}$ according to Eq. (12). $K_{\mathrm{e} 2} / K_{\mathrm{e} 1}$ is the ratio of the $K_{\mathrm{e}}$ produced by organisms at time $t_{2}$ over the $K_{\mathrm{e}}$ produced by organisms at an earlier time $t_{1}$ in the absence of sampling or other outside interference. The $y$-axis shows how the $K_{\mathrm{e}}$ at time $t_{2}$ is affected by taking a $56 \mathrm{ml}$ sample at time $t_{1}$ (approximating the effect of taking four $14 \mathrm{ml}$ samples prior to time $t_{2}$ ); a value of 1.1, for example, indicates that $K_{\mathrm{e}}$ at time $t_{2}$ is 1.1 times greater if prior sampling takes place than if it does not. Total volume of large bottle is $1070 \mathrm{ml}$; that of small bottle is a third as great. $F_{\mathrm{e} 1}=0.65$. With these parameters, $f$ is 0.92 for the large bottle, 0.76 for the small one. (C) Concentration of liquid or gas in a standard bottle relative to initial concentration, as a function of the number of $1 \mathrm{ml}$ liquid samples withdrawn, estimated using Eq. (13) for $F_{\mathrm{s}(\mathrm{i})}=0.65$. The relation is presented for bottles of 2 total volumes common in catalogs. A $1 \mathrm{ml}$ sample represents a $F_{\mathrm{a}}$ of 0.0063 for the larger bottle but 0.026 for the smaller one in experimental bottles must be independent of $F_{v(1)}$ in the bottles (and of correlates of $F_{V(1)}$ such as partitioning effects). We introduced our analysis using studies of the production of chemicals like DMS that originate entirely from the liquid phase, but such studies are just one application. Our analytical solution can be applied, for example, to studies of degradation of chemicals by aquatic organisms if degradation per unit liquid volume can be assumed independent of $F_{V(l)}$.
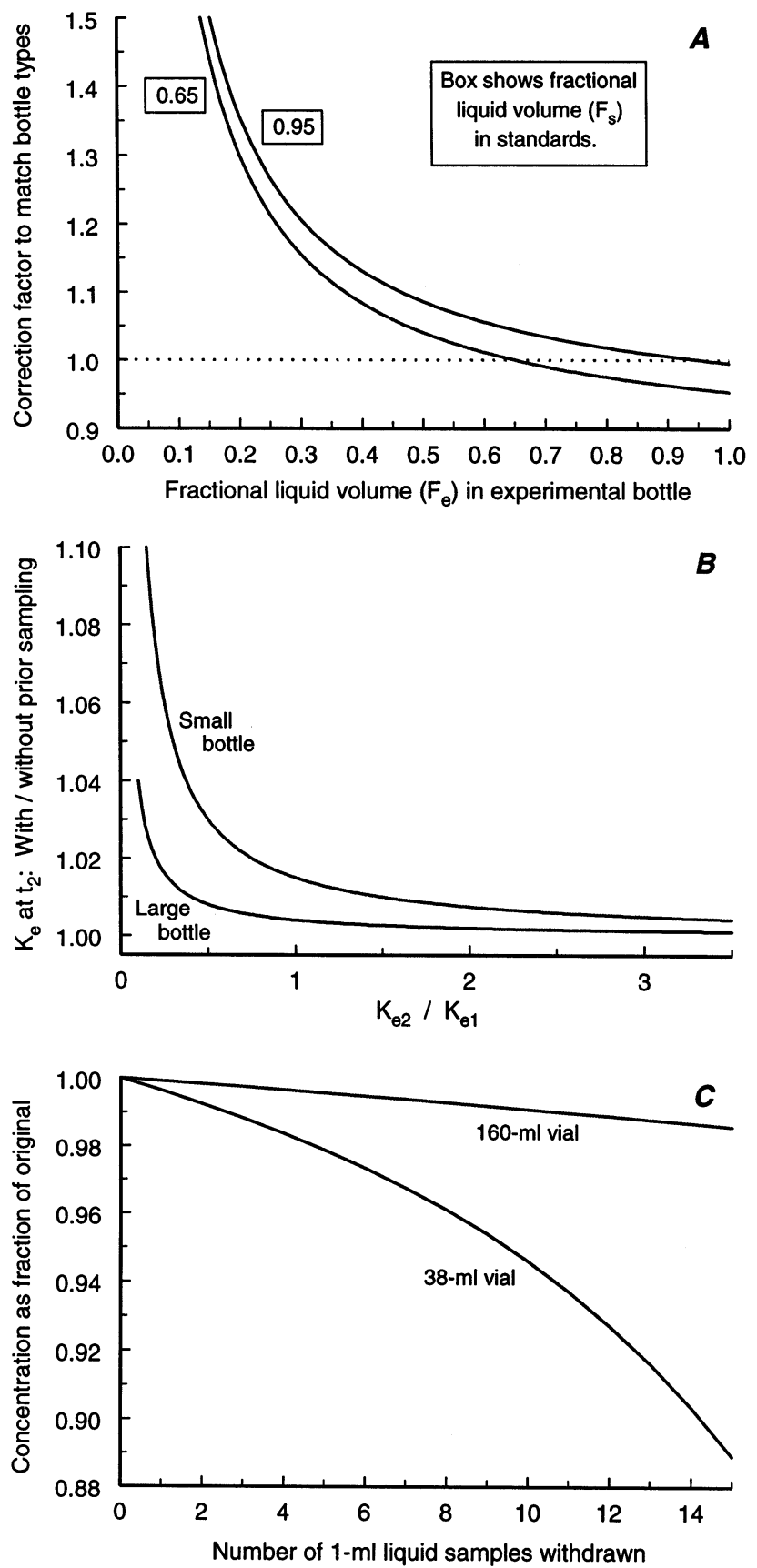


\section{Problem 2: Effect of sampling from an experimental bottle on later values in the bottle}

A second problem in studies of volatile compounds is that sample removal from cultures in experimental bottles distorts concentrations in later samples from those bottles: sampling effects confound biological effects. Symbols useful for analysis of this problem are defined in Table 1C. Suppose that in a study of DMS formation, the time is $t_{2}$ and $F_{V(1)}$ in the experimental bottle, before sampling, is $F_{\mathrm{e} 2}$. Consider 2 scenarios for reaching this point: scenario $\mathrm{N}$ (no prior sampling) and scenario $\mathrm{P}$ (prior sampling). Under scenario $\mathrm{N}$, the bottle was initially filled, at time $t_{0}$, with DMS-free culture to the level $F_{V(1)}=F_{\mathrm{e} 2}$, and it is being sampled for the first time at $t_{2}$. The prevailing $K_{\mathrm{e}}$ will be, quite simply, the DMS level that the organisms create between $t_{0}$ and $t_{2}$. Under scenario $P$, the bottle was previously sampled; it was filled at $t_{0}$ with DMS-free culture at higher liquid level $F_{\mathrm{V}(1)}=F_{\mathrm{e} 1}$, and sampling at an earlier time $t_{1}$ brought $F_{\mathrm{V}(1)}$ to $F_{\mathrm{e} 2}$. Under this scenario, the $K_{\mathrm{e}}$ prevailing at $t_{2}$ is susceptible to influence by the prior sampling. Problem 1 concerned the correct quantification of prevailing $K_{\mathrm{e}}$. Problem 2 concerns the meaning of the prevailing $K_{\mathrm{e}}$.

Continue the assumptions made in Problem 1 (e.g., a homogeneous culture, $K_{\mathrm{e}}$ independent of $\left.F_{V(1)}\right)$. The prevailing $K_{\mathrm{e}}$ at $t_{2}$ under scenario $\mathrm{N}$ is $K_{\mathrm{e} 2}$. What is the prevailing $K_{\mathrm{e}}$ at $t_{2}$ under scenario $\mathrm{P}$ ? Under scenario $\mathrm{P}$, at $t_{1}$, just before sample removal, $n$ was $K_{\mathrm{e} 1} F_{\mathrm{e} 1} V_{\mathrm{T}}$ (from Eq. 1). To calculate $n$ at $t_{2}$, we first need to subtract the quantity of DMS removed by sampling at $t_{1}$; this quantity is the product of the sample volume at $t_{1}\left[(1-f) F_{\mathrm{e} 1} V_{\mathrm{T}}\right]$ and the liquid concentration at $t_{1}$ (Eq. 6 evaluated for $F_{\mathrm{e} 1}$ and $K_{\mathrm{e} 1}$ ). Then we need to add the change in $n$ between $t_{1}$ and $t_{2}$, which is calculated as the product of prevailing liquid volume, $f F_{\mathrm{e} 1} V_{\mathrm{T}}\left(=F_{\mathrm{e} 2} V_{\mathrm{T}}\right)$, and the difference between $K_{\mathrm{e} 2}$ and $K_{\mathrm{e} 1}$. Thus, for scenario $\mathrm{P}$,

$$
n \text { at } t_{2}=K_{\mathrm{el}} F_{\mathrm{el}} V_{\mathrm{T}}-\frac{(1-f) F_{\mathrm{el}}^{2} V_{\mathrm{T}} K_{\mathrm{el}} L}{1+(L-1) F_{\mathrm{el}}}+f F_{\mathrm{el}} V_{\mathrm{T}}\left(K_{\mathrm{e} 2}-K_{\mathrm{el}}\right)
$$

Dividing Eq. (11) by $f F_{\mathrm{e} 1} V_{\mathrm{T}}\left(=F_{\mathrm{e} 2} V_{\mathrm{T}}\right)$ gives the prevailing $K_{\mathrm{e}}$ at $t_{2}$ under scenario P. Thus:

$\frac{K_{\mathrm{e}} \text { at } t_{2} \text { under scenario } \mathrm{P}}{K_{\mathrm{e}} \text { at } t_{2} \text { under scenario } \mathrm{N}}=\frac{K_{\mathrm{e} 2}-K_{\mathrm{e} 1}}{K_{\mathrm{e} 2}}+\frac{K_{\mathrm{e} 1}}{K_{\mathrm{e} 2}}\left(\frac{1-F_{\mathrm{e} 1}+F_{\mathrm{e} 1} f L}{f-f F_{\mathrm{e} 1}+F_{\mathrm{e} 1} f L}\right)$

Note that the final ratio (in parentheses) depends entirely on experimental design, and once it is fixed, an equation dependent on biologically produced changes in $K_{\mathrm{e}}$ results. Fig. 1B shows examples. Note that the $K_{\mathrm{e}}$ prevailing at $t_{2}$ is always larger with prior sampling than without; this is because liquid sampling leaves behind gas-phase DMS that was produced by liquid no longer present and that becomes 'misattrib- uted' to the smaller liquid volume remaining. Note also that the effect of prior sampling tends to be small when the amount of DMS in the bottle is rising $\left(K_{\mathrm{e} 2} / K_{\mathrm{e} 1}>1\right)$, but substantial signal-averaging and distortion can occur in a falling-DMS situation $\left(K_{\mathrm{e} 2} / K_{\mathrm{e} 1}<1\right)$. The effect of prior sampling becomes smaller as the final ratio (in parentheses) in Eq. (12) is made smaller; thus, designing experiments with relatively large $F_{\mathrm{e} 1}$ and $f$ reduces distortion. Informed choices of $F_{\mathrm{e} 1}$ and $f$ can reduce errors to inconsequential levels, at least in rising-DMS situations.

\section{Problem 3: Effect of sampling from a standard on later accuracy of the standard}

A third problem is the effect of removing liquid samples from standards on the accuracy of the standards. This problem is actually a special case of Problem 2. Assume no loss of gas in removing samples, and refer to Table 1D for parameter definitions. If each sample removes an assay volume $F_{\mathrm{a}} V_{\mathrm{T}}$, and $z$ is the number of samples withdrawn, a first-approximation approach is to model the cumulative impact of sample removal at each $z$ as equivalent to withdrawal of a volume $z F_{\mathrm{a}} V_{\mathrm{T}}$ of the original equilibrated liquid. This approximation overestimates changes in accuracy of the standard by ignoring iterative reductions in the [DMS] of liquid withdrawn, but the assumption of no gas loss causes underestimation if gas in fact can escape when liquid samples are taken.

When a standard of nominal concentration $K_{\mathrm{s}}$ is prepared, its initial liquid concentration at equilibrium, $C_{\mathrm{s}(\mathrm{i})}$, is given by Eq. (6) with $F_{\mathrm{V}(\mathrm{l})}=F_{\mathrm{s}(\mathrm{i})}$ and $K=$ $K_{\mathrm{s}}$. The total quantity of DMS in the standard bottle after removal of $z$ samples, $n_{z \prime}$ can be calculated from Eq. (11) by recognizing that for the present problem, $K_{\mathrm{e} 2}=K_{\mathrm{e} 1}=K_{\mathrm{s}}, F_{\mathrm{e} 1}=F_{\mathrm{s}(\mathrm{i})}$, and $f=\left(F_{\mathrm{s}(\mathrm{i})}-z F_{\mathrm{a}}\right) / F_{\mathrm{s}(\mathrm{i})}$. Actual $K$ after $z$ samples, $K_{z \prime}$ is $n_{z}$ divided by the liquid volume at that point, $\left(F_{\mathrm{s}(i)}-z F_{\mathrm{a}}\right) V_{\mathrm{T}}$. The concentration of the liquid after $z$ samples, $C_{\mathrm{s}(z)}$, is calculated from Eq. (6) with $K=K_{z}$ and $F_{V(1)}=\left(F_{\mathrm{s}(\mathrm{i})}-z F_{\mathrm{a}}\right)$, and:

$$
\frac{C_{\mathrm{s}(z)}}{C_{\mathrm{s}(\mathrm{i})}}=\frac{1+F_{\mathrm{s}(\mathrm{i})} L-F_{\mathrm{s}(\mathrm{i})}-z F_{\mathrm{a}} L}{1+F_{\mathrm{s}(\mathrm{i})} L-F_{\mathrm{s}(\mathrm{i})}-z F_{\mathrm{a}} L+z F_{\mathrm{a}}}
$$

The equation describes how liquid [DMS] changes in relation to its initial value as liquid is removed from a standard of fixed nominal (initial) concentration $K_{\mathrm{s}}$. Headspace [DMS] changes identically in relation to initial headspace [DMS] as liquid is removed $\left[C_{1}=\right.$ $L C_{\mathrm{g}}$ ]. Fig. $1 \mathrm{C}$ shows that multiple sampling can seriously jeopardize the integrity of a standard of relatively low initial volume. 


\section{DISCUSSION}

Several experimental designs exist for establishing a time-series while taking liquid samples during incubation studies of microbial processing of volatile compounds such as DMS. From the viewpoint of biology, we believe that the inherently most sound design is repeated sampling from individual bottles without replacement. However, this design presents unique problems of potential sampling-induced artifacts because when sampling changes liquid:gas volume ratios within bottles, it in itself induces redistribution and concentration alterations of volatile compounds. The potential sampling-induced problems of the design need not be a worry if the problems are addressed analytically. This paper presents equations that permit informed choice of volumetric parameters (bottle sizes, sample sizes, etc.) so that artifacts are constrained within defined, investigator-determined limits. The equations also permit correction of many artifacts by calculation.

In multiple-sample experiments, volumetric parameters are at least as consequential as chemical analytical methods, microbial enumeration procedures, and many other details of technique that are already viewed as essential considerations. Full details of volumetric design should be required information in research reports.

\section{Effects of liquid:gas partitioning behavior}

Our consistent use of DMS as a focal compound in our derivations has the advantage of simplicity but may tend to obscure the fact that the specific, temperature-dependent partitioning behavior of each compound is a highly relevant parameter. The Ostwald coefficient $L$, which expresses the concentration in the liquid phase as a ratio of the concentration in the gas phase at equilibrium, appears in all of our analytical solutions. Eqs. (10), (12), \& (13) show that within the range of $L$ we consider $(L=2-20)$, decreases in $L$ act to increase all the effects of sampling we discuss. Thus, for some chemicals - and even for DMS at some temperatures - the magnitudes of problems introduced by sampling could be substantially greater than shown in Fig. 1 (where $L=11$ is assumed). The phenomena discussed in this paper arise from the 2-phase nature of volatile compounds. In the range of $L$ discussed, increases in $L$ represent a shift toward a 1-phase system.

\section{Homogeneous versus nonhomogeneous cultures}

We have focused on homogeneous cultures because most studies of the dynamics of DMS production and degradation in the literature are of homogeneous or quasi-homogeneous material and the mathematics are tractable. The opposite extreme would be a culture in which the source (or sink) of DMS (or other compound) is a point source that remains entirely in the experimental bottle during sampling (e.g., a fish that causes DMS liberation). To illustrate modeling of a point source, consider Problem 1. Our solution, Eq. (10), for homogeneous cultures rests on conservation of $K_{\mathrm{e}}$ regardless of $F_{V(1)}$ in an experimental bottle. For a point source, the quantity conserved is $n$, the total amount of DMS produced in the bottle. Thus, $I_{\text {e(adj) }}$ for a point source is obtained as:

$$
I_{\mathrm{e}(\mathrm{adj})}=I_{\mathrm{e}}\left[\frac{1+(L-1) F_{\mathrm{e}}}{1+(L-1) F_{\mathrm{s}}}\right]
$$

instead of from Eq. (10). Some of the mathematics for a point source are not so tractable. Also, a pure pointsource model may often not be realistic because even if a point source is responsible for some addition or subtraction of DMS (or other compound of interest), homogeneously distributed organisms that add or subtract (e.g., bacteria) are likely to be present. Experimental designs that permit the homogeneous model to be applied are desirable.

Acknowledgements. Thanks to Gary King for invaluable editorial advice. This work was completed while the authors received support from Woods Hole Oceanographic Institution Sea Grant NA90AADSG480 and Michigan Sea Grant College Program NA76RG0133. WHOI Contribution No. 9554.

\section{LITERATURE CITED}

Andreae MO, Crutzen PJ (1997) Atmospheric aerosols: biogeochemical sources and role in atmospheric chemistry. Science 276:1052-1058

Bak F, Finster K, Rothfuß F (1992) Formation of dimethylsulfide and methanethiol from methoxylated aromatic compounds and inorganic sulfide by newly isolated anaerobic bacteria. Arch Microbiol 157:529-534

Clever HL, Battino R (1975) The solubility of gases in liquids. In: Dack MRJ (ed) Techniques of chemistry, Vol 8, Part 1. Wiley, New York, p 379-441

Dacey JWH, Wakeham SG (1986) Oceanic dimethylsulfide: production during zooplankton grazing on phytoplankton. Science 233:1314-1316

Dacey JWH, Wakeham SG, Howes BL (1984) Henry's law constants for dimethylsulfide in freshwater and seawater. Geophys Res Lett 11:991-994

Daly KL, DiTullio GR (1996) Particulate dimethylsulfoniopropionate removal and dimethylsulfide production by zooplankton in the southern ocean. In: Kiene RP, Visscher PT, Keller MD, Kirst GO (eds) Biological and environmental chemistry of DMSP and related sulfonium compounds. Plenum, New York, p 223-238

Gerrard W (1980) Gas solubilities. Pergamon, New York Hill RW, White BA, Cottrell MT, Dacey JWH (1998) Virusmediated total release of dimethylsulfoniopropionate from 
marine phytoplankton: a potential climate process. Aquat Microb Ecol 14:1-6

Kiene RP (1996) Turnover of dissolved DMSP in estuarine and shelf waters of the northern Gulf of Mexico. In: Kiene RP, Visscher PT, Keller MD, Kirst GO (eds) Biological and environmental chemistry of DMSP and related sulfonium compounds. Plenum, New York, p 337-348

Kiene RP, Service SK (1991) Decomposition of dissolved DMSP and DMS in estuarine waters: dependence on temperature and substrate concentration. Mar Ecol Prog Ser 76:1-11

Ledyard KM, Dacey JWH (1996) Microbial cycling of DMSP and DMS in coastal and oligotrophic seawater. Limnol Oceanogr 41:33-40

Mackay D, Shiu WY (1981) A critical review of Henry's law constants for chemicals of environmental interest. J Phys Chem Ref Data 10:1175-1199

Malin G, Kirst GO (1997) Algal production of dimethyl sulfide and its atmospheric role. J Phycol 33:889-896

Malin G, Wilson WH, Bratbak G, Liss PS, Mann NH (1998)

Editorial responsibility: Gary King,

Walpole, Maine, USA
Elevated production of dimethylsulfide resulting from viral infection of cultures of Phaeocystis pouchetii. Limnol Oceanogr 43:1389-1393

Przyjazny A, Janicki W, Chrzanowski W, Staszewski R (1983) Headspace gas chromatographic determination of distribution coefficients of selected organosulfur compounds and their dependence on some parameters. J Chromatogr 280:249-260

Stefels J, van Boekel WHM (1993) Production of DMS from dissolved DMSP in axenic cultures of the marine phytoplankton species Phaeocystis sp. Mar Ecol Prog Ser 97:11-18

Visscher PT, Kiene RP, Taylor BF (1994) Demethylation and cleavage of dimethylsulfoniopropionate in marine intertidal sediments. FEMS Microbiol Ecol 14:179-190

Wolfe GV, Steinke M (1996) Grazing-activated production of dimethyl sulfide (DMS) by two clones of Emiliania huxleyi. Limnol Oceanogr 41:1151-1160

Wolfe GV, Steinke M, Kirst GO (1997) Grazing-activated chemical defence in a unicellular marine alga. Nature $387: 894-897$

Submitted: August 31, 2000; Accepted: February 22, 2001 Proofs received from author(s): May 15, 2001 